\title{
VCSEL-Based Optical Frequency Combs Expansion Induced by Polarized Optical Injection
}

\author{
Ana Quirce, Cristina de Dios, Angel Valle, and Pablo Acedo, Member, IEEE
}

\begin{abstract}
The present theoretical work studies optical frequency combs (OFCs) associated with both linear polarizations of a gain switching vertical-cavity surface-emitting laser (VCSEL) subject to optical injection of arbitrary polarization. The overall OFC, formed by the two orthogonally polarized sub-combs, can have an expanded optical span in agreement with experiments reported by Prior et al. We show that the maximum optical span is obtained for a certain angle between the linearly polarized optical injection and the direction of emission of the main mode of the free-running VCSEL. We find that this angle decreases as the injection strength increases. We also characterize the maximum expansion of the optical span of the overall comb. For VCSELs with large birefringence splitting the maximum expansion is the optical span of the free-running OFC, while for small birefringence splitting VCSELs the maximum expansion is the value of the birefringence splitting.
\end{abstract}

Index Terms-Semiconductor lasers, Vertical-Cavity SurfaceEmitting Lasers, gain switching, optical frequency comb generator, optical injection, polarization.

1

\section{INTRODUCTION}

Optical Frequency Combs (OFCs) consist of a series of evenly spaced discrete spectral components that maintain high spectral coherence [1]. OFCs find applications in fields such as metrology, spectroscopy, optical arbitrary waveform generation, $\mathrm{THz}$ generation, microwave photonics or optical communications [1]-[5]. One way of generating OFCs is by using semiconductor lasers. These systems are compact with low energy consumption, cost and complexity. Such OFCs can contribute to take out of the laboratory technologies such as dual-combs [3] or optical processing [6]. Different technologies have been used to generate these OFCs. For instance, mode-locking [7], electro-optical modulation [8], and gain-switching [9] of semiconductor lasers produce good quality OFCs.

Gain-switching (GS) consists on the periodic RF large signal modulation of the current applied to a laser diode

Manuscript received September, 2018. This work was funded by the Ministerio de Economía y Competitividad (MINECO/FEDER, UE), Spain under project TEC2015-65212-C3-1-P. The work of A. Quirce was supported by the Fonds Wetenschappelijk for the Post-Doctoral Fellowship.

A. Quirce is with the Faculty of Engineering Sciences, Vrije Universiteit Brussel, 1050, Brussels, Belgium. e-mail: aquirce@b-phot.org

C. de Dios, and P. Acedo are with the Univ. Carlos III , Avenida de la Universidad, 20, 28911, Leganés, Madrid, Spain. e-mail: cdios@ing.uc3m.es

A. Valle is with Instituto de Física de Cantabria (CSIC-Univ. Cantabria), Avda. Los Castros s/n, E39005, Santander, Spain. e-mail: valle@ifca.unican.es; pesquerl@ifca.unican.es

${ }^{1}$ Copyright (c) 2015 IEEE. Personal use of this material is permitted. However, permission to use this material for any other purposes must be obtained from the IEEE by sending a request to pubs-permissions@ieee.org. to generate a regular pulse train. GS of single-mode semiconductor lasers offer combs with good tunability characteristics, and high efficiency and correlation between optical modes [9], [10]. OFCs using different types of commercial single-mode semiconductor lasers have been generated. Edgeemitting lasers like Distributed feedback (DFB) or Discrete Mode Lasers (DML) [9], [11] and Vertical-Cavity SurfaceEmitting Lasers (VCSEL) [10], [12], [13] have been used.

VCSELs are single-longitudinal mode semiconductor lasers with several advantages over edge-emitters like on-wafer testing capability, lower energy consumption, reduced manufacturing costs, and circular output beam [14], [15]. These properties make them appropriate for mass production and for OFC generation [10]. Single-transverse mode VCSELs can emit in two linear polarization modes with orthogonal directions [15]. Polarization switching (PS) between these modes can be found when changing the temperature or bias current applied to the VCSEL [15]-[17]. This unique behaviour gives VCSELs an extra degree of freedom with respect to edgeemitters for which linearly polarized emission is usually found. Another way for obtaining PS is by applying external optical injection [18]-[21]. PS has been usually obtained for the case of orthogonal optical injection in which linearly polarized light is injected orthogonally to the linear polarization of the main mode of the free-running VCSEL [18]-[21]. PS has also been observed when both previous polarizations are parallel (parallel optical injection) [22], and when the injected light has arbitrary polarization [23].

Due to the special polarization properties of these devices dual-polarization VCSEL based OFCs can be generated, opening perspectives for polarization-sensitive sensing and multicarrier optical sources for polarization-division multiplexing optical communications. A simple way to obtain these OFCs is by gain-switching VCSELs: it has been experimentally [10] and theoretically shown [13] that two orthogonally polarized OFCs are generated in such a way that combine to produce a wider overall optical comb, although one of the OFCs has smaller power than the other. Optical injection has also been used experimentally to improve the characteristics of VCSEL-based gain switching OFCs [24]. Linearly polarized optical injection can produce an overall comb with enhanced optical span in which both subcombs have comparable span and power, [24]. Also an overall comb with single linear polarization that is parallel or orthogonal was obtained for parallel and orthogonal optical injection, respectively [24].

In this work we perform a theoretical analysis of OFCs obtained when a gain-switched VCSEL is subject to arbitrary optical injection. Our model is based on the rate equations 
of the spin-flip model extended to consider the general case of ellipticaly polarized injected light. Our work mainly aims at explaining the experimental results of [24]. We obtain similar results to those of [24] when we consider parallel and orthogonally linearly polarized optical injection and also more general cases, like linearly polarized light with an arbitrary angle or elliptically polarized light. We show that expanded OFCs, in which both subcombs have similar span and power can be obtained, in agreement with [24]. Our work goes beyond the findings of [24] by predicting that the maximum span is obtained for an specific angle which value depends on the injection strength. We will also discuss the limits of this method of OFC expansion.

The structure of this paper is organized as follows. We present in section II the theoretical model. Free-running OFCs are described in section III. In section IV we describe the effect of optical injection with arbitrary polarization on OFCs. Section V is devoted to analyze and discuss our results. Finally, in section VI we summarize our results.

\section{THEORETICAL MODEL}

Optical injection effects on the polarization dynamics of gain-switched VCSELs are theoretically studied by using the spin-flip model (SFM) [25]-[27]. Usual versions of SFM consider linear carrier recombination. However good quality OFCs, with large optical span and large carrier to noise ratio, are obtained when modulating sinusoidally the current applied to the VCSEL with large amplitudes. Under these conditions nonlinear carrier recombination terms are preferred for simulation [13], [28] because the carrier number can depart significantly with respect to its threshold value in such a way that linearization of the carrier recombination term around threshold is no longer a good approximation [13].

In the SFM model the dynamical evolution of the linear polarization modes of a single-transverse mode VCSEL is described. The linearly polarized complex e-fields in the $x$ and $y$ directions are $E_{x}$ and $E_{y}$, respectively. There are two carrier variables. The first one is $D=\left(N-N_{t}\right) /\left(N_{t h}-N_{t}\right)$ where $N, N_{t h}$, and $N_{t}$ are the carrier number, carrier number at threshold, and at transparency, respectively. The second one is $n$, that is the difference of the carriers associated with the spinup and spin-down levels [26]. The SFM equations modelling VCSELs subject to a sinusoidal modulation of the applied current and to optical injection of arbitrary polarization are [13], [23]:

$$
\begin{aligned}
\frac{d E_{x}}{d t}= & -\left(\kappa+\gamma_{a}\right) E_{x}-i\left(\kappa \alpha+\gamma_{p}\right) E_{x} \\
& +\kappa(1+i \alpha)\left(D E_{x}+i n E_{y}\right)+\kappa E_{i n j, x} e^{i\left(2 \pi \nu_{i n j} t+\delta\right)} \\
& +\left(\sqrt{\frac{R_{+}}{2}} \xi_{+}(t)+\sqrt{\frac{R_{-}}{2}} \xi_{-}(t)\right) \\
\frac{d E_{y}}{d t}= & -\left(\kappa-\gamma_{a}\right) E_{y}-i\left(\kappa \alpha-\gamma_{p}\right) E_{y} \\
& +\kappa(1+i \alpha)\left(D E_{y}-i n E_{x}\right)+\kappa E_{i n j, y} e^{i 2 \pi \nu_{i n j} t} \\
& +i\left(\sqrt{\frac{R_{-}}{2}} \xi_{-}(t)-\sqrt{\frac{R_{+}}{2}} \xi_{+}(t)\right)
\end{aligned}
$$

$$
\begin{aligned}
\frac{d D}{d t}= & \frac{I}{e \Delta N_{t}}-R(D)-\gamma\left[D\left(\left|E_{x}\right|^{2}+\left|E_{y}\right|^{2}\right)\right. \\
& \left.+i n\left(E_{y} E_{x}^{*}-E_{x} E_{y}^{*}\right)\right] \\
\frac{d n}{d t}= & -\gamma_{s} n-\gamma\left[n\left(\left|E_{x}\right|^{2}+\left|E_{y}\right|^{2}\right)\right. \\
& \left.+i D\left(E_{y} E_{x}^{*}-E_{x} E_{y}^{*}\right)\right]
\end{aligned}
$$

where

$$
\begin{gathered}
R_{ \pm}=\beta_{S F} \gamma\left[(D \pm n)+\frac{G_{N} N_{t}}{2 \kappa}\right] \\
R(D)=A\left(D+D_{t}\right)+B\left(D+D_{t}\right)^{2}+C\left(D+D_{t}\right)^{3} \\
I(t)=I_{0}\left(1+m \cos \left(2 \pi f_{m} t\right)\right)
\end{gathered}
$$

, $D_{t}=N_{t} / \Delta N_{t}$, and $\Delta N_{t}=N_{t h}-N_{t}$. The functions $R(D)$ and $I(t)$ correspond to the nonlinear carrier recombination, and the applied sinusoidal bias current, respectively. This current is characterized by the following parameters: $I_{0}$ is the bias current, $m$ is the modulation index, and $f_{m}$ is the modulation frequency. The meaning of the rest of the VCSEL parameters with their corresponding numerical values can be found in Table I. Gaussian white noises, $\xi_{+}(t)$ and $\xi_{-}(t)$ are considered to simulate the effect of spontaneous emission noise [27]. Parameters in Table I correspond to the 1550$\mathrm{nm}$ wavelength VCSEL (Vertilas) characterized in [13] by following the method developed in [26], [29] complemented by measurements of the turn-on delay time, as in [30], in order to obtain the $A, B$, and $C$ parameters.

Optical injection terms are included in e-field rate equations in order to consider both the azimuth and ellipticity of the injected light [23]. In these equations $E_{i n j, x}$ and $E_{i n j, y}$ are the amplitudes of the injected light in $x$ and $y$ polarizations, respectively. $\nu_{i n j}$ is the difference between the frequency of the optical injection and the frequency intermediate between those of the $x$-polarized and $y$-polarized modes of the freerunning VCSEL, $\nu_{x}$ and $\nu_{y}$, given by $2 \pi \nu_{x}=\alpha \gamma_{a}-\gamma_{p}$ and $2 \pi \nu_{y}=\gamma_{p}-\alpha \gamma_{a}$, respectively. In this work we give our results using the frequency detuning with respect to the frequency of the $x$-polarized mode, that is $\nu_{i}=\nu_{i n j}-\nu_{x}$. We also shift the frequency of optical spectra in a quantity given by $\left(\gamma_{p}-\right.$ $\left.\alpha \gamma_{a}\right) /(2 \pi)$ in order to have a zero frequency that corresponds to the frequency of the $x$-polarized mode. A phase term, $\delta$, is included in the injection term of the equation for $E_{x}$ in order to simulate an arbitrary polarization as in [23]. This is done without loss of generality by setting the phase of the injected $y$-e-field to zero, $\delta_{y}=0$, and the phase of the $x$-efield to $\delta, \delta_{x}=\delta$, in order to simplify the equations, so $\delta$ is the phase difference between $x$ - and $y$ - components of the injected e-field [23]. This definition is appropriate when the linear dichroism is positive in such a way that the dominant mode of the solitary VCSEL is linearly polarized parallel to the $y$-direction [23]. The state of polarization of the optical injection is completely characterized by $\delta$ and by an auxiliary angle, $\theta_{p}$, such that $E_{i n j, x}=E_{i} \sin \theta_{p}, E_{i n j, y}=E_{i} \cos \theta_{p}$, 
where $E_{i}$ is the amplitude of the optical injection [23]. We will use as optical injection parameters $E_{i}, \nu_{i}, \theta_{p}$ and $\delta$. The values of $\delta$ and $\theta_{p}$ are such that $-\pi / 2 \leq \delta \leq \pi / 2$, and $0 \leq \theta_{p} \leq$ $\pi / 2$. $\theta_{p}=0\left(\theta_{p}=\pi / 2\right)$ corresponds to linearly polarized emission parallel to the $y(x)$ direction. Linearly polarized light forming an angle $\theta_{p}$ with the $y$-axis is characterized by $\left(\theta_{p}, \delta\right)=\left(\theta_{p}, 0\right)$. Different degrees of elliptical polarization can be achieved by changing the value of $\delta$. For instance, right/left circularly polarized light is obtained for $\left(\theta_{p}, \delta\right)=$ $(\pi / 4, \pm \pi / 2)$.

TABLE I

VCSEL'S PARAMETER VALUES

\begin{tabular}{cll}
\hline Parameter & Meaning & Value \\
\hline$\kappa$ & Field decay rate & $36 \mathrm{~ns}^{-1}$ \\
$\gamma_{a}$ & Linear dichroism & $1.4 \mathrm{~ns}^{-1}$ \\
$\gamma_{p}$ & Linear birefringence & $106 \mathrm{~ns}^{-1}$ \\
$\alpha$ & Linewidth enhancement factor & 2.1 \\
$\beta_{S F}$ & Spontaneous emission parameter & $5.4 \cdot 10^{-4}$ \\
$\gamma$ & Decay rate of D & $2.8 \mathrm{~ns}^{-1}$ \\
$G_{N}$ & Differential gain & $3.37 \cdot 10^{4} \mathrm{~s}^{-1}$ \\
$N_{t}$ & Carrier number at transparency & $1.49 \cdot 10^{7}$ \\
$N_{t h}$ & Carrier number at threshold & $1.70 \cdot 10^{7}$ \\
$\gamma_{s}$ & Spin-flip relaxation rate & $1000 \mathrm{~ns}^{-1}$ \\
$A$ & Nonradiative coefficient & $2.1 \cdot 10^{7} \mathrm{~s}^{-1}$ \\
$B$ & Radiative coefficient & $6.0 \cdot 10^{7} \mathrm{~s}^{-1}$ \\
$C$ & Auger coefficient & $7 \cdot 10^{6} \mathrm{~s}^{-1}$ \\
\hline
\end{tabular}

\section{Free-RunNing OpticAl Frequency Combs}

In this section we analyze the dynamics of the free-running VCSEL, that is without external optical injection. We perform a numerical integration of Eqs.(1)-(4) in order to obtain the optical spectra. In our simulations we use a $0.01 \mathrm{ps}$ integration time step, a $2.5 \mathrm{ps}$ sampling time, and an average over 20 temporal windows of $40.96 \mathrm{~ns}$ duration. We choose a value of the bias current well above the threshold current, $I_{0}=11.5$ $\mathrm{mA}=4.4 I_{t h}$. Continuous-wave $(\mathrm{CW})$ operation $(m=0)$ is illustrated in Fig. 1(a) in which optical spectrum corresponding to the total power and both linear polarizations are shown. The frequency separation between the peaks corresponding to the two linear polarizations is $34 \mathrm{GHz} . y$-linear polarization mode dominates the emission (because $\gamma_{a}>0$ ) with the largest optical frequency $\left(\gamma_{p}>0\right)$.

The formation of optical frequency combs for several values of the modulation frequency is shown in the remaining parts of Fig. 1. An effective frequency range for comb generation from VCSELs using GS technique exists around the relaxation oscillation frequency [13]. This range corresponds to the excitation of only the first spike of the relaxation oscillations in such a way that there is not a complete extinction of the stimulated emission between successive pulses. We have chosen a modulation index of $m=1.3$ for which good agreement with experiments [10] was found [13]. Unless stated otherwise we will maintain $m=1.3$ and $I_{0}=11.5 \mathrm{~mA}$ in this work. In Fig. 1(b) we show optical spectra obtained for $f_{m}=5 \mathrm{GHz}$. Both $x$ - and $y$-polarization modes exhibit OFCs centered around their corresponding $\mathrm{CW}$ frequencies. The $x$-polarization mode is characterized by an OFC with larger optical power than in $\mathrm{CW}$ regime due the excitation
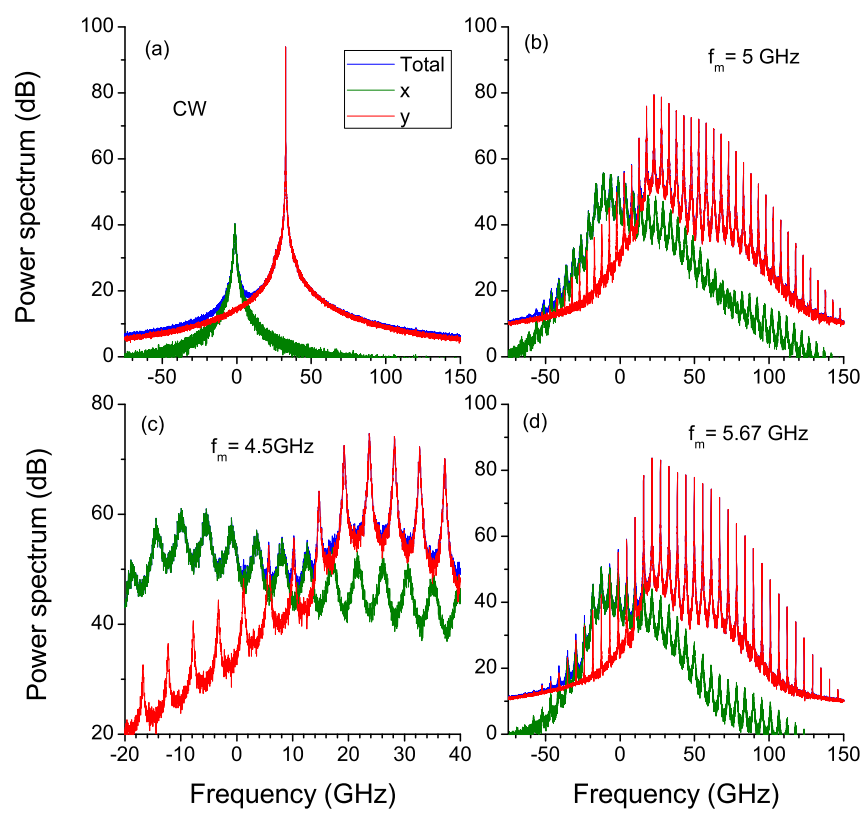

Fig. 1. Optical spectra of the total output, $x$-polarization, and $y$-polarization modes for (a) $\mathrm{CW}$ operation (b) $f_{m}=5 \mathrm{GHz}$ (c) $f_{m}=4.5 \mathrm{GHz}$, and (d) $f_{m}=5.67 \mathrm{GHz}$.

of pulses in the suppresed polarization that occurs in the gainswitching regime [10], [13]. The power of the $x$-polarized OFC is smaller than that of the $y$-polarized OFC, as explained in [13]. A way of characterizing the quality of the OFC is given by the Carrier to Noise Ratio (CNR) that we define as the difference, in $\mathrm{dB}$, between the maximum of the optical spectrum and the minimum of its next valley. We will refer to $\mathrm{CNR}, \mathrm{CNR}_{x}$, and $\mathrm{CNR}_{y}$ to the values of carrier-to-noise ratio for OFCs corresponding to the total, $x$-polarized and $y$ polarized emission, respectively. $x$-polarized OFC has smaller value of $\mathrm{CNR}\left(\mathrm{CNR}_{x}=12 \mathrm{~dB}\right)$ than that of $y$-polarized $\mathrm{OFC}$ $\left(\mathrm{CNR}_{y}=30 \mathrm{~dB}\right)$ because of the strongest effect of spontaneous emission noise in the formation of $x$-polarized pulses [13].

One characteristic of linearly polarized OFCs that can be seen in Fig. 1(b) and Fig. 1(c) is that peaks in the $x$-polarized optical spectrum do not appear at the same frequencies as those of $y$-polarized spectrum. This happens because peaks in each OFC appear centered in their CW frequency and separated by $f_{m}$. So in general matching between peaks frequencies does not happen because the frequency separation between linear polarizations, approximately given by $\gamma_{p} / \pi$, and $f_{m}$ can take any value. This unmatching causes the appearance of not equally spaced peaks in the spectrum of the total output in regions in which both polarized OFCs have comparable powers, as it can be seen in Fig. 1(c) in the frequency region around $10 \mathrm{GHz}$. A simple way of obtaining matching between both lineary polarized OFCs is by choosing a value of $f_{m}$ such that an integer multiple of $f_{m}$ equals the frequency separation between the peaks corresponding to the two linear polarizations in the $\mathrm{CW}$ spectrum. For instance by choosing 
$6 f_{m}=34 \mathrm{GHz}$ we get $f_{m}=5.67 \mathrm{GHz}$. The situation for this value of $f_{m}$ is shown in Fig. 1(d) in which matching is apparent and so regularly spaced peaks in the spectrum of the total power are obtained. This value of the modulation frequency will be used in our simulations from now on in order to have more regular spectra for which the discussion of optical injection effects will be made in a more clear way.

\section{EFFEct of Optical InJeCtion with ARbitrary POLARIZATION ON OFCS}

In this section we analyze the effect of the injection of light with arbitrary polarization on the OFCs formation. Fig. 2 shows optical spectra obtained for parallel optical injection, that is when injecting linearly polarized light parallel to the $y$-direction $\left(\theta_{p}=0\right)$. Fig. 2(a) illustrates the situation when the frequency of the optical injection is between the two main peaks of the $y$-polarized OFC in Fig. 1(d), $\nu_{i}=24$ $\mathrm{GHz}$, and $E_{i}=0.07$. Optical spectrum of the $y$-polarized OFC has much more lines because two subcombs coexist in the spectrum. The first one is similar to the free-running OFC shown in Fig. 1(d), although with smaller power and CNR. The second one is centered at the injection frequency with a frequency separation between lines of $f_{m}$. A zoom of Fig. 2(a) is shown in Fig. 2(b) to better illustrate this situation. $x$-polarized OFC has also traces of this double comb structure in the region around $50 \mathrm{GHz}$ but the comb quality deteriorates with respect to that in Fig. 1(d) because CNR in Fig. 2(a) decreases. Fig. 2(c) shows spectra when $E_{i}$ increases to 0.5 . Only the $y$-polarized comb centered at $\nu_{i}$ survives. This OFC has locked to the optical injection in such a way that its power and CNR are larger than those of the corresponding free-running OFC. Emission in the $x$-direction is very weak without comb formation. These results are similar to those obtained in [24] under parallel optical injection. The $y$-polarized OFC behavior is similar to that described in a single-polarization mode gain-switched DML subject to optical injection [28].

The situation changes for orthogonal optical injection, that is when injecting linearly polarized light parallel to the $x$-direction $\left(\theta_{p}=\pi / 2\right)$. In Fig. 3 we show optical spectra when $\nu_{i}=-4 \mathrm{GHz}$, that corresponds to an injection frequency between two consecutive main peaks of the $x$-polarized OFC in Fig. 1(d). Fig. 3(a) shows that when $E_{i}=0.07$ optical injection mainly affects the $x$-polarized OFC while there is almost no effect on the $y$-polarized OFC (it is very similar to that in Fig. 1(d)). We now identify two subcombs in the $x$-polarized optical spectrum: one is similar to the freerunning $x$-polarized OFC of Fig. 1(d), and the other is centered at $\nu_{i}$ with a frequency spacing of $f_{m}$. The $x$-polarized OFC has much more power than in Fig. 1(d) in such a way that the spectrum of the total power is enhanced over a wider frequency range but without the regular $f_{m}$ frequency spacing of Fig. 1(d) as it can be seen in Fig. 3(b), in which a zoom corresponding to Fig. 3(a) has been represented. As in Fig. 2 , an increase of the injected power leads to an $x$-polarized OFC locked to the optical injection, as it is shown in Fig. 3(c) and its corresponding zoom of Fig. 3(d). At these large values

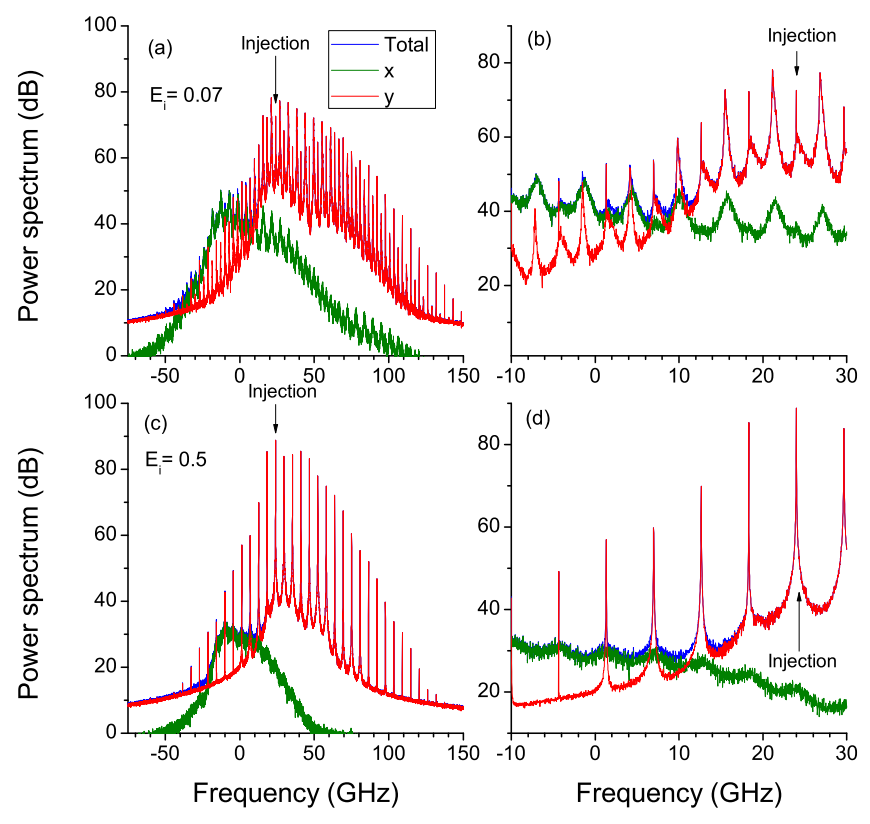

Fig. 2. Optical spectra of the total output, $x$-polarization, and $y$-polarization modes under $y$-polarized optical injection for (a) $E_{i}=0.07$ (c) $E_{i}=0.5$. (b) and (d) are zooms of (a), and (c), respectively. In this figure $\theta_{p}=0$, $\delta=0$, and $\nu_{i}=24 \mathrm{GHz}$

of $E_{i}$ the emission in the $y$-direction is very weak without clear OFC formation. The selection of the $x$-polarized OFC is similar to that described in experiments under orthogonal optical injection [24].

A way of obtaining regularly spaced OFCs when using optical injection for any value of $E_{i}$ is by injecting at a $\nu_{i}$ value at which both free-running polarized OFC have a peak. This is shown in Fig. 4(a) and Fig. 4(d) for $y$-polarized and $x$-polarized optical injection, respectively. This figure has been obtained for $\nu_{i}=-12.6 \mathrm{GHz}$, and $E_{i}=0.07$, that is the same injection strength of Fig. 2(a) and Fig 3(a). Fig. 4(a) shows that both OFCs are very similar to those in Fig. 1(d). However, more interestingly, there is a very significantly change in the spectra of Fig. 4(d). This figure shows that $x$-polarized optical injection can increase the width of the spectrum of the total power because of the excitation of the $x$-polarized OFC with large power. Also the spectrum of the total power corresponds to a regular OFC with a frequency spacing of $f_{m}$. Enhancement of the frequency width of the OFC due to optical injection can be characterized by the 10 -dB spectral width, $S W_{10}$. We define it as the maximum frequency separation between peaks with values larger than 10- $\mathrm{dB}$ below the absolute maximum of the optical spectrum. That enhancement is clear because $S W_{10}$ increases from 45.4 GHz in Fig. 1(d) to $68 \mathrm{GHz}$ in Fig. 4(d).

We now compare the temporal evolutions of the power of both linear polarizations for the cases of Fig. 1(d) and Fig. 4(d) in order to explain the frequency enhancement observed in this last figure. Fig. 5(a) and Fig. 5(b) show time traces for 

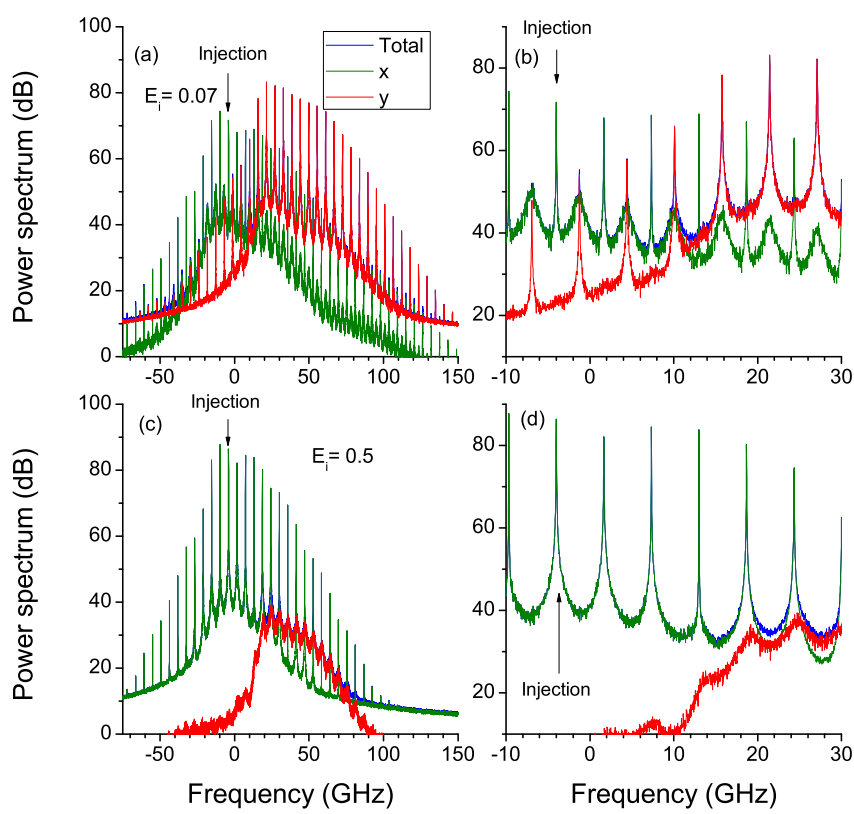

Fig. 3. Optical spectra of the total output, $x$-polarization, and $y$-polarization modes under $x$-polarized optical injection for (a) $E_{i}=0.07$ (c) $E_{i}=0.5$. (b) and (d) are zooms of (a), and (c), respectively. In this figure $\theta_{p}=\pi / 2$, $\delta=0$, and $\nu_{i}=-4 \mathrm{GHz}$

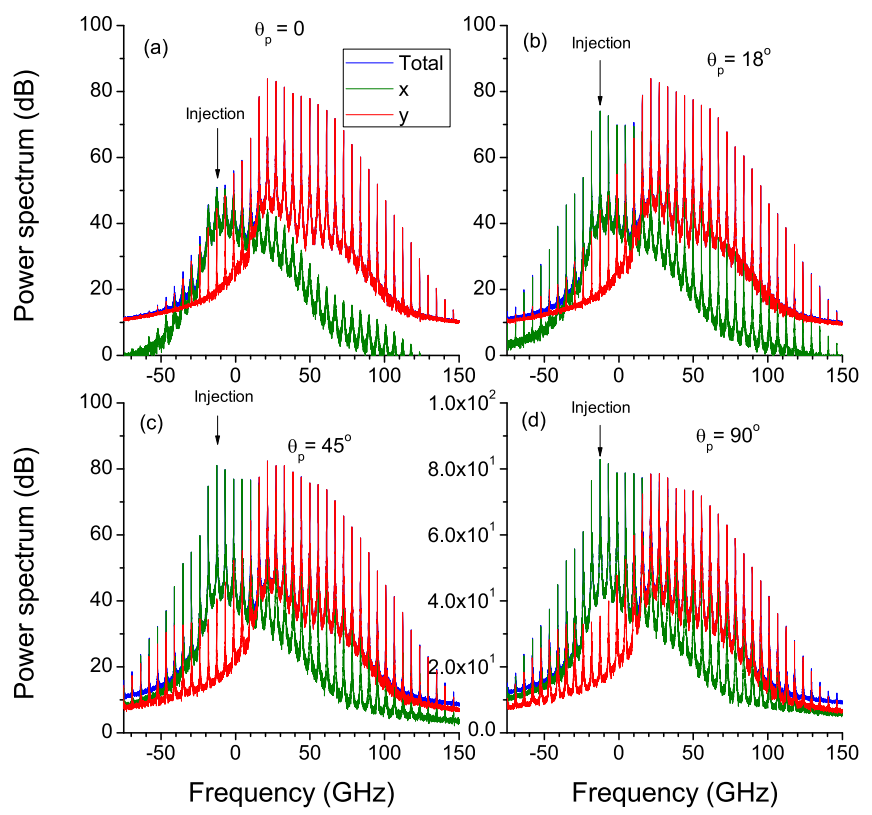

Fig. 4. Optical spectra of the total output, $x$-polarization, and $y$-polarization modes under linearly polarized optical injection forming different angles with the $y$ - direction: (a) $\theta_{p}=0$, (b) $\theta_{p}=\pi / 10$, (c) $\theta_{p}=\pi / 4$, and (d) $\theta_{p}=\pi / 2$. In this figure $\delta=0, E_{i}=0.07$, and $\nu_{i}=-12.6 \mathrm{GHz}$
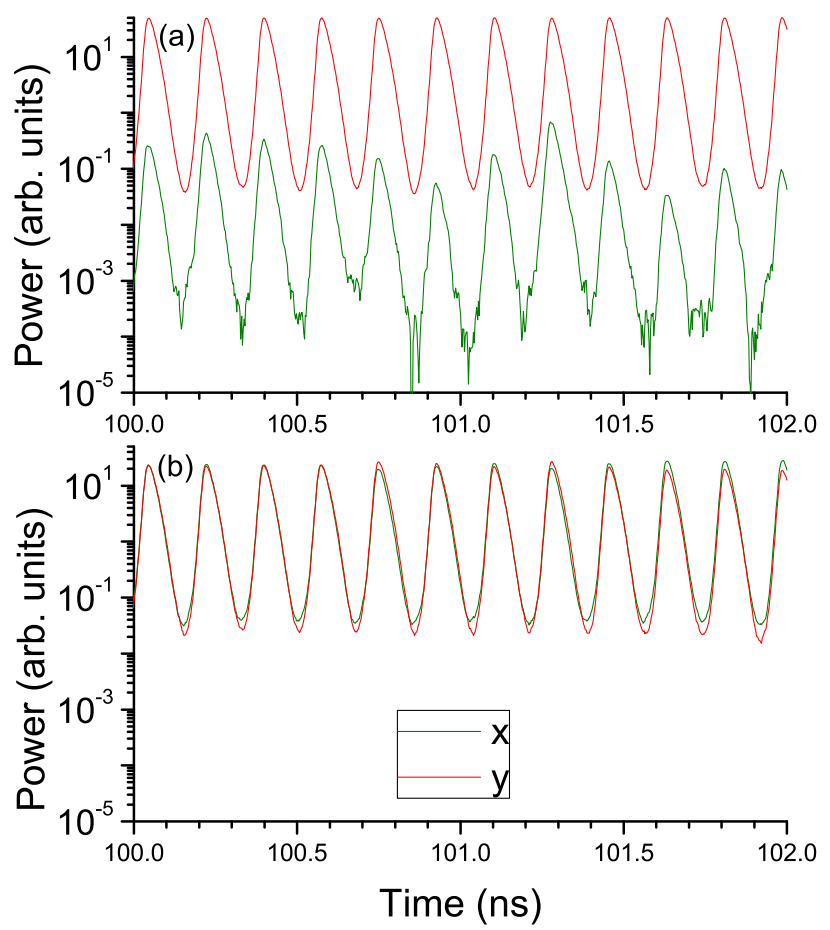

Fig. 5. Time traces of the power of $x$ (green line) and $y$-polarization (red line) modes for (a) a free-running VCSEL, and (b) a VCSEL subject to linearly polarized optical injection parallel to the $x$-direction with $E_{i}=0.07$, and $\nu_{i}=-12.6 \mathrm{GHz}$.

the cases included in Fig. 1(d) and Fig. 4(d), respectively. A vertical logarithmic scale has been chosen in order to show clearly the effect of spontaneous emission noise. Fig. 5(a) shows a $y$-polarized regular pulse train with $5.67 \mathrm{GHz}$ repetition frequency. Pulse minima are well above the level at which spontaneous emission noise dominates the evolution, that is around $10^{-4}$. This is not the situation for the $x$-polarized pulse train because spontaneous emission noise has a large effect. This noise causes a strong irregularity in the height and time of emission of the pulses. So coherence between pulses is lost and the $x$-polarized OFC in Fig. 1(d) has a low value of $\mathrm{CNR}_{x}$. Fig. 5(b) shows that optical injection linearly polarized parallel to the $x$-direction is able to increase minimum values of $x$-polarized power well above the level of spontaneous emission noise dominance. So a regular $x$-polarized pulse train is also obtained with the corresponding excitation of a good quality $x$-polarized OFC and subsequent frequency enhancement of the total power OFC, as it is shown in Fig. 4(d).

We now analyze the effect of the angle $\theta_{p}$ between the direction of a linearly polarized optical injection and the $y$-axis, that is the direction of emission of the free-running VCSEL. Up to now we have seen the effect when $\theta_{p}=0$ and $\pi / 2$ in Fig. 4(a), and Fig. 4(d), respectively. Fig. 4(b) and Fig. 4(c) show optical spectra when $\theta_{p}$ is $0.1 \pi$ and $\pi / 4$, respectively. Excitation of the $x$-polarized OFC can be 
obtained even for a small value of $\theta_{p}$, as it is shown in Fig. 4(b): $\mathrm{CNR}_{x}$ increases from $13.6 \mathrm{~dB}$ to $35.7 \mathrm{~dB}$ when going from Fig. 4(a) to Fig. 4(b). Fig. 4(c) shows that when $\theta_{p}$ increases to $\pi / 4$ the $x$-polarized OFC increases in power with a value of $\mathrm{CNR}_{x}, 40.8 \mathrm{~dB}$, similar to that obtained for the orthogonal optical injection illustrated in Fig. 4(d), 42.3 dB. In summary, Fig. 4 indicates that there is a wide range of $\theta_{p}$ values for which frequency enhancement of OFC induced by linearly polarized optical injection can be obtained. The effect of $\theta_{p}$ on the overall comb width will be analyzed in the next section.

We now study the effect of injecting elliptically polarized light on the formation of OFCs by comparing with previous results. Fig. 6 shows optical spectra obtained under different ellipticity conditions of the optical injection. In Fig. 6(a) results corresponding to elliptically polarized light with an instantaneous ellipticity, $\chi=18^{\circ}$ are plotted, where $\chi$ is defined by $\sin 2 \chi=\sin 2 \theta_{p} \sin \delta$ [23]. Comparison with corresponding results for linearly polarized injection of Fig. 4(b) shows similar spectra with similar values of $\mathrm{SW}_{10}$ and $\mathrm{CNR}_{y}$ and a slightly larger $\mathrm{CNR}_{x}$ value, $1 \mathrm{~dB}$, for linearly polarized injection. Injection of right circular light, illustrated in Fig. 6(b), gives also similar results to those obtained under corresponding linearly polarized injection (Fig. 4(c)). Fig. 6(c) shows results obtained when increasing $\chi$ to $72^{\circ}$. Comparison between Fig. 6(b) and Fig. 6(c) shows that increasing $\chi$ excites $x$-polarized OFC while the power of $y$-polarized OFC decreases. Fig. 6(d) illustrates spectra obtained for injection of left circular light under the same conditions to those of Fig. 6(b). Changing from right to left circularly polarized light scarcely affects optical spectrum as it can be seen by comparing Fig. 6(b) and Fig. 6(d). These results seem to indicate that the effect of ellipticity of the injected beam on OFCs formation is not important, as it will be shown in the next section.

Injection-locked states can be found where $x$ and $y$-polarized subcombs are both injection-locked to the $\mathrm{CW}$ external optical injection. This happens for instance in cases illustrated in Fig. 4(b) and 4(c). Situations in which only one subcomb is injection-locked to the external light with no coherence between the two subcombs also occur (see Fig. 2(d) and Fig. 3(d)). For elliptically polarized optical injection we also find injection-locked states for $x$ and $y$-polarized subcombs, for instance in Fig. 6(b). In this case, around the considered detuning $\nu_{i}=-12.6 \mathrm{GHz}$, we find locking if $13 \mathrm{GHz} \leq \nu_{i} \leq-12 \mathrm{GHz}$, so the locking range is $1 \mathrm{GHz}$. The locking range increases as $E_{i}$ increases. Optical spectrum corresponding to each linear polarization changes within this locking range in such a way that excitation of the $x$-polarized subcomb increases as we approach the borders of the locking interval. The optimum spectrum obtained in Fig. 6(b) only occurs at selected (discrete) values of GS frequencies, $f_{m}$, as discussed in Fig. 1(d). The ranges for $f_{m}$ values that satisfy the condition of Fig. 1(d) are small, around $20 \mathrm{MHz}$ for the case of Fig. 6(b).

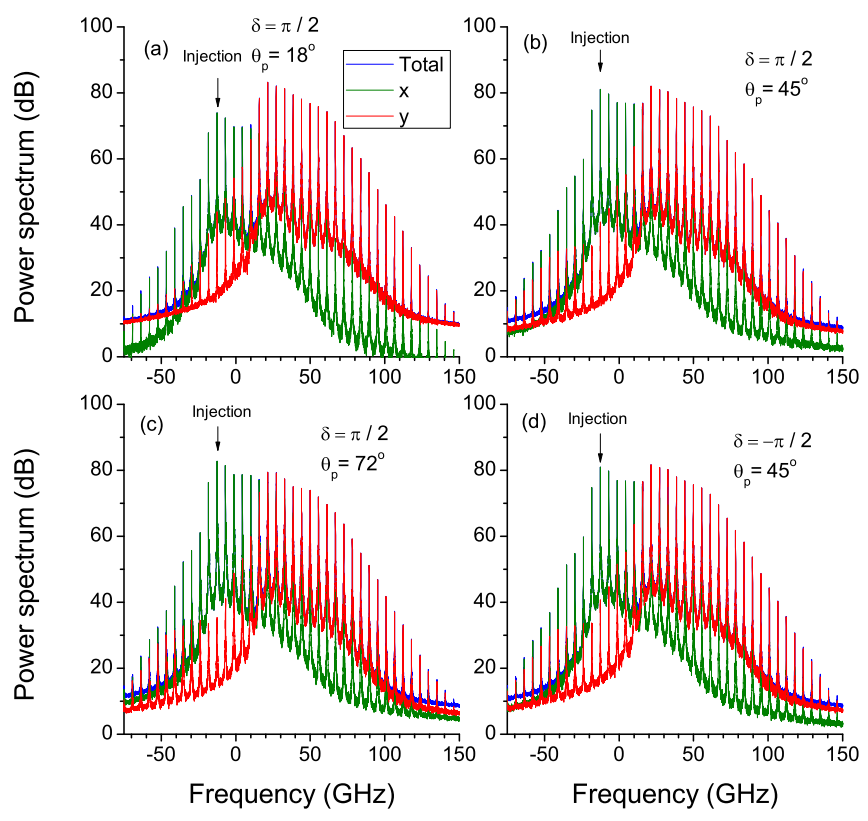

Fig. 6. Optical spectra of the total output, $x$-polarization, and $y$-polarization modes under elliptically polarized optical injection: (a) $\theta_{p}=0.1 \pi, \delta=\pi / 2$, (b) $\theta_{p}=\pi / 4, \delta=\pi / 2$, (c) $\theta_{p}=0.4 \pi, \delta=\pi / 2$, and (d) $\theta_{p}=\pi / 4$, $\delta=-\pi / 2$. In this figure, $E_{i}=0.07$, and $\nu_{i}=-12.6 \mathrm{GHz}$.

\section{ANALYSIS AND Discussion}

In this section we analyze the effect of optical injection on the quality of generated OFCs. We will also discuss strategies for optimizing the enhancement of the optical span induced by the optical injection. First we calculate the values of $\mathrm{CNR}_{x}$, $\mathrm{CNR}_{y}$, and $\mathrm{SW}_{10}$ when the VCSEL is subject to linearly polarized optical injection. Fig. 7 shows these quantities as a function of the angle between the injected electrical field and the $y$-direction, $\theta_{p}$, for several values of $E_{i}$. Fig. 7(a) shows that $\mathrm{CNR}_{x}$ increases with $\theta_{p}$, as was illustrated in Fig. 4, because the projection of the injected field over the $x$-direction increases. Also this projection increases as $E_{i}$ increases, so $\mathrm{CNR}_{x}$ monotonously increases with $E_{i}$.

Fig. 7(b) shows that when $E_{i}$ is small $\mathrm{CNR}_{y}$ does not change with $\theta_{p}$ nor $E_{i}$. This corresponds to the small effect of the optical injection on an already well formed $y$-polarized $\mathrm{OFC}$, as it is also illustrated in Fig. 4. However, Fig. 7(b) shows that when $E_{i}$ increases to $0.14, \mathrm{CNR}_{y}$ begins to decrease for $\theta_{p}>30^{\circ}$. This happens because the projection of the injected field over the $x$-direction has increased in such a way that there is only an $x$-polarized OFC, locked to the optical injection, with a negligible emission in the $y$-direction. This is similar to the situation illustrated in Fig. 3(c).

Fig. 7(c) shows that $S W_{10}$ does not change with $\theta_{p}$ when this angle is small, being $45.4 \mathrm{GHz}$ the value of $S W_{10}$. The projection of the injected field over the $x$-direction is not enough to excite the $x$-polarized OFC above the $10-\mathrm{dB}$ level. So the $S W_{10}$ value corresponds to that obtained for the free running OFC, that we term as $S W_{10, f}$. The value obtained 

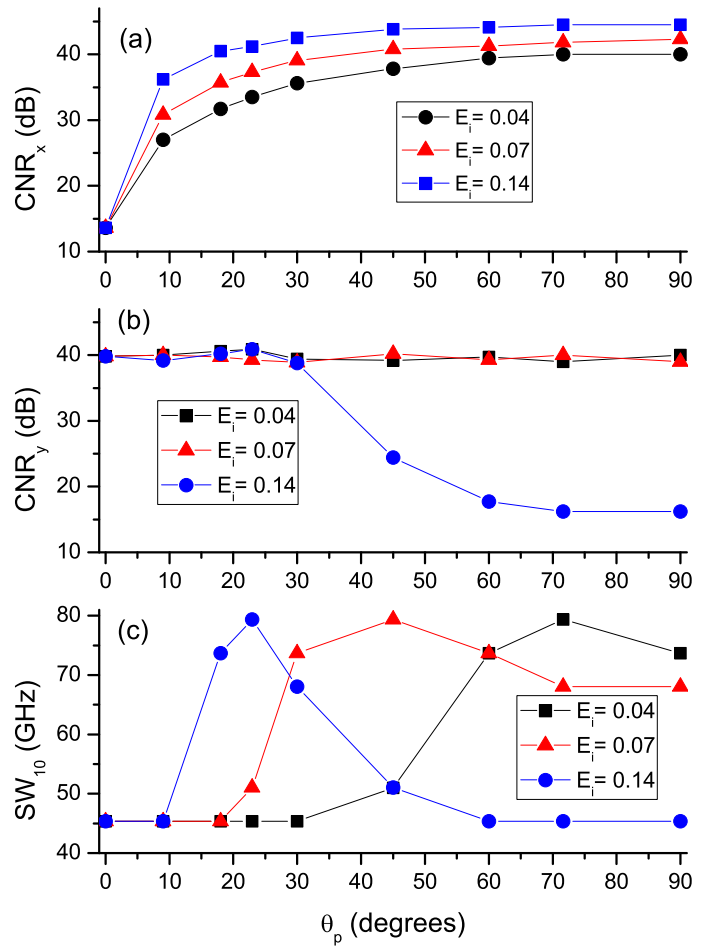

Fig. 7. (a) $\mathrm{CNR}_{x}$, (b) $\mathrm{CNR}_{y}$, and (c) $10-\mathrm{dB}$ Spectral width as a function of $\theta_{p}$ for several values of $E_{i}$. In this figure, $\delta=0$, and $\nu_{i}=-12.6 \mathrm{GHz}$.

for $S W_{10, f}$ from Fig. 1(d) is precisely $45.4 \mathrm{GHz}$. We also observe in Fig. 7(c) that there is an optimum $\theta_{p}$ for which $S W_{10}$ is maximum. This maximum value is $79.4 \mathrm{GHz}$ no matter the value of $E_{i}$. The frequency enhancement is 34 $\mathrm{GHz}$, that is precisely the frequency difference between both linear polarizations in the $\mathrm{CW}$ spectrum, given basically by $\gamma_{p} / \pi$. Fig. 7(c) also shows that the $\theta_{p}$ value for which $S W_{10}$ is maximum decreases when $E_{i}$ increases. This maximum is obtained when full excitation of the $x$-polarized OFC occurs while the $y$-OFC still remains excited. This happens when there is an appreciable projection of the injected field over the $x$-direction and this projection is obtained for smaller values of $\theta_{p}$ when $E_{i}$ increases. The decrease of $S W_{10}$ when $\theta_{p}$ increases is due to the decrease in power of the $y$-polarized OFC. This decrease is slow for low values of $E_{i}$. However for $E_{i}=0.14$ that decrease is more abrupt, like in Fig. 7(b), because it occurs through dissappearance of the $y-\mathrm{OFC}$ due to injection locking of the $x-\mathrm{OFC}$.

In order to check that elliptical optical injection plays a similar role to that of linearly polarized light we have performed simulations similar to those of Fig. 7 but with different values of $\delta: \pi / 2$ and $\pi / 4$. Results, not shown, are very close to those obtained with $\delta=0$. This is of interest to obtain experimentally our results because it should be easy to find a configuration of the polarization controller for which frequency enhancement of the OFC occurs.

An strategy for maximizing the OFC frequency enhancement is to choose a VCSEL with larger value of $\gamma_{p}$. Huge enhancement of VCSEL birefringence can be achieved by the technique of introducing an anisotropic mechanical strain [31]. VCSELs with birefringence splitting above $250 \mathrm{GHz}$ $\left(\gamma_{p}>785 \mathrm{~ns}^{-1}\right)$ have been already demonstrated [32]. We show in Fig. 8(a) optical spectra for a VCSEL subject to linearly polarized optical injection in which $\gamma_{p}$ is $160 \mathrm{~ns}^{-1}$. The value of $S W_{10}$ is $96 \mathrm{GHz}$, larger than those obtained in Fig. 7. However the limits of this enhancement can be appreciated because of the development of a valley in the spectrum of the total ouput around $30 \mathrm{GHz}$. As $\gamma_{p}$ increases both orthogonally polarized combs can be excited but their larger frequency separation makes this valley to appear. Lines around this valley are still above the 10-dB level and still contribute to $S W_{10}$. However, further increase of $\gamma_{p}$ will make some lines in the valley fall below the $10-\mathrm{dB}$ level, as it is illustrated in Fig. 8(b) in which results for $\gamma_{p}=250 \mathrm{~ns}^{-1}$ are plotted. The maximum value of $S W_{10}$ is obtained for the case illustrated in Fig. 8(a) because the value at the valley is close to the 10-dB level. This case illustrates the situation in which $\gamma_{p} / \pi>S W_{10, f}$. For this case $S W_{10} \sim 2 S W_{10, f}$ because the spectral width has two similar contributions coming from each polarized comb which value is approximately $S W_{10, f}$. For cases analyzed in Fig. 7, in which $\gamma_{p} / \pi<S W_{10, f}$, the maximum frequency width is smaller than that of Fig. 8 (a) and is given by $S W_{10} \sim S W_{10, f}+\gamma_{p} / \pi$. Summarizing, for VCSELs with large birefringence splitting the maximum frequency enhancement is the spectral width of the freerunning OFC, $S W_{10, f}$, while for small birefringence splitting VCSELs the frequency enhacement is precisely the value of this splitting, $\gamma_{p} / \pi$. Another strategy for obtaining combs with wider frequency range is to use strong optical injection regime to shift relaxation oscillation frequency to higher value, as suggested in a theoretical study on single-mode edge emitting lasers [33]. This idea applied to VCSELs will be the subject of future work.

\section{CONCLUSions}

Optical frequency combs generated by GS find applications in optical communications [9], in absorption spectroscopy [34], [35], and in sub-THz generation [4]. Injection locking of GS DM lasers shows a flexible comb covering continuous frequency ranges [9]. This is particularly important in the context of flexible OFCs for the flexible/re-configurable optical communication systems. 10-dB spectral width, $S W_{10}$, larger than $80 \mathrm{GHz}$ can be easily achieved in injection-locked GS DML [9], [11]. In this work we have shown that appropriate injection locking in GS VCSELs can increase $S W_{10}$ to values comparable to those obtained with GS DMLs. This increase is important for enhancing performance of OFCs generated by GS VCSELs for the previously mentioned applications.

To conclude, in this work we have analyzed theoretically optical frequency combs that appear in a gain switching VCSEL subject to optical injection of arbitrary polarization. We have shown that the overall comb is composed by two orthogonally 


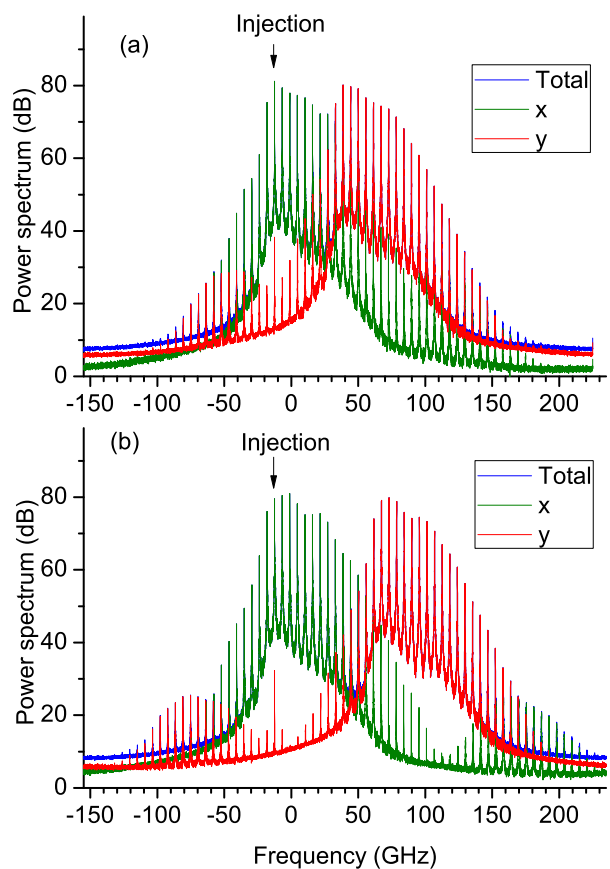

Fig. 8. Optical spectra of the total output, $x$-polarization, and $y$-polarization modes under linearly polarized optical injection. (a) $\gamma_{p}=160 \mathrm{~ns}^{-1}$, (b) $\gamma_{p}=250 \mathrm{~ns}^{-1}$. In this figure, $\delta=0, \theta_{p}=40^{\circ}, E_{i}=0.07$, and $\nu_{i}=-12.6$ GHz.

polarized sub-combs that can have comparable span and power under certain optical injection conditions. These conditions are such that optical injection e-field must have a significant projection over the direction associated to the suppressed linear polarization mode of the solitary VCSEL. Linear optical injection forming a $\theta_{p}$ angle with this direction can induce an expanded optical span. Also elliptically polarized optical injection have similar effects to those of the linear optical injection. Our results are in agreement with experiments reported in [24]. We have shown that the maximum optical span is obtained for a value of $\theta_{p}$ that decreases as the strength of the optical injection increases. We have characterized the maximum expansion of the optical span of the overall comb. For VCSELs with large birefringence splitting the maximum frequency enhancement is the optical span of the free-running comb, while for small birefringence splitting the maximum frequency enhancement is the birefringence splitting value.

\section{REFERENCES}

[1] N. R. Newbury, "Searching for applications with a fine-tooth comb," Nature Photonics, vol. 5, no. 4, pp. 186-188, 2011.

[2] I. Coddington, N. Newbury, and W. Swann, "Dual-comb spectroscopy," Optica, vol. 3, no. 4, p. 414, apr 2016.

[3] P. Martin-Mateos, M. Ruiz-Llata, J. Posada-Roman, and P. Acedo, "Dual-Comb Architecture for Fast Spectroscopic Measurements and Spectral Characterization," IEEE Photonics Technology Letters, vol. 27, no. 12, pp. 1309-1312, jun 2015.
[4] A. R. Criado, C. de Dios, E. Prior, G. H. Dohler, S. Preu, S. Malzer, H. Lu, A. C. Gossard, P. Acedo, C. de Dios, E. Prior, G. H. Döhler, S. Preu, S. Malzer, H. Lu, A. C. Gossard, and P. Acedo, "ContinuousWave Sub-THz Photonic Generation With Ultra-Narrow Linewidth, Ultra-High Resolution, Full Frequency Range Coverage and High LongTerm Frequency Stability," IEEE Transactions on Terahertz Science and Technology, vol. 3, no. 4, pp. 461-471, jul 2013.

[5] M. Imran, P. M. Anandarajah, A. Kaszubowska-Anandarajah, N. Sambo, and L. Potí, "A survey of optical carrier generation techniques for terabit capacity elastic optical networks," IEEE Communications Surveys \& Tutorials, vol. 20, no. 1, pp. 211-263, 2018.

[6] T. P. McKenna, J. H. Kalkavage, M. D. Sharp, and T. R. Clark, "Wideband Photonic Compressive Sampling System," Journal of Lightwave Technology, vol. 34, no. 11, pp. 2848-2855, jun 2016.

[7] J. Davila-Rodriguez, K. Bagnell, and P. J. Delfyett, "Frequency stability of a $10 \mathrm{ghz}$ optical frequency comb from a semiconductor-based modelocked laser with an intracavity 10,000 finesse etalon," Optics letters, vol. 38, no. 18, pp. 3665-3668, 2013.

[8] V. Torres-Company and A. M. Weiner, "Optical frequency comb technology for ultra-broadband radio-frequency photonics," Laser \& Photonics Reviews, vol. 8, no. 3, pp. 368-393, 2014.

[9] P. Anandarajah, R. Maher, Y. Xu, S. Latkowski, J. O'carroll, S. Murdoch, R. Phelan, J. O'Gorman, and L. Barry, "Generation of coherent multicarrier signals by gain switching of discrete mode lasers," IEEE Photonics Journal, vol. 3, no. 1, pp. 112-122, 2011.

[10] E. Prior, C. De Dios, M. Ortsiefer, P. Meissner, and P. Acedo, "Understanding vcsel-based gain switching optical frequency combs: Experimental study of polarization dynamics," Journal of Lightwave Technology, vol. 33, no. 22, pp. 4572-4579, 2015.

[11] A. Rosado, A. Pérez-Serrano, J. M. G. Tijero, Á. Valle, L. Pesquera, and I. Esquivias, "Experimental study of optical frequency comb generation in gain-switched semiconductor lasers," Optics \& Laser Technology, vol. 108, pp. 542-550, 2018.

[12] E. Prior, C. de Dios, A. R. Criado, M. Ortsiefer, P. Meissner, and P. Acedo, "Expansion of vcsel-based optical frequency combs in the subthz span: Comparison of non-linear techniques," J. Lightwave Technol., vol. 34, no. 17, pp. 4135-4142, Sep 2016.

[13] A. Quirce, C. de Dios Fernandez, A. Valle, L. Pesquera, and P. Acedo, "Polarization dynamics in vcsel-based gain switching optical frequency combs," Journal of Lightwave Technology, vol. 36, no. 10, pp. 17981806, 2018.

[14] F. Koyama, "Recent advances of VCSEL photonics," Journal of Lightwave Technology, vol. 24, no. 12, pp. 4502-4513, 2006.

[15] R. Michalzik, VCSELs: fundamentals, technology and applications of vertical-cavity surface-emitting lasers. Springer, 2012, vol. 166.

[16] G. Verschaffelt, K. Panajotov, J. Albert, B. Nagler, M. Peeters, J. Danckaert, I. Veretennicoff, and H. Thienpont, "Polarisation switching in vertical-cavity surface-emitting lasers: from experimental observations to applications," OPTOELECTRONICS REVIEW, no. 3, pp. 257-268, 2001.

[17] K. Panajotov, J. Danckaert, G. Verschaffelt, M. Peeters, B. Nagler, J. Albert, B. Ryvkin, H. Thienpont, and I. Veretennicoff, "Polarization behavior of vertical-cavity surface-emitting lasers: Experiments, models and applications," in AIP Conference Proceedings, vol. 560, no. 1. AIP, 2001, pp. 403-417.

[18] Z. G. Pan, S. Jiang, M. Dagenais, R. A. Morgan, K. Kojima, M. T. Asom, R. E. Leibenguth, G. D. Guth, and M. W. Focht, "Optical injection induced polarization bistability in vertical-cavity surface-emitting lasers," Applied physics letters, vol. 63, no. 22, pp. 2999-3001, 1993.

[19] B. Ryvkin, K. Panajotov, E. Avrutin, I. Veretennicoff, and H. Thienpont, "Optical-injection-induced polarization switching in polarizationbistable vertical-cavity surface-emitting lasers," Journal of applied physics, vol. 96, no. 11, pp. 6002-6007, 2004.

[20] M. Sciamanna and K. Panajotov, "Two-mode injection locking in vertical-cavity surface-emitting lasers," Optics letters, vol. 30, no. 21, pp. 2903-2905, 2005.

[21] I. Gatare, M. Sciamanna, M. Nizette, and K. Panajotov, "Bifurcation to polarization switching and locking in vertical-cavity surface-emitting lasers with optical injection," Physical Review A, vol. 76, no. 3, p. 031803, 2007.

[22] A. Quirce, P. Pérez, A. Popp, Ángel Valle, L. Pesquera, Y. Hong, H. Thienpont, and K. Panajotov, "Polarization switching and injection locking in vertical-cavity surface-emitting lasers subject to parallel optical injection," Opt. Lett., vol. 41, no. 11, pp. 2664-2667, Jun 2016.

[23] R. Al-Seyab, K. Schires, A. Hurtado, I. D. Henning, and M. J. Adams, "Dynamics of vesels subject to optical injection of arbitrary polariza- 
tion," IEEE Journal of Selected Topics in Quantum Electronics, vol. 19, no. 4, pp. 1700512-1700 512, 2013.

[24] E. Prior, C. de Dios, R. Criado, M. Ortsiefer, P. Meissner, and P. Acedo, "Dynamics of dual-polarization vcsel-based optical frequency combs under optical injection locking," Optics letters, vol. 41, no. 17, pp. 40834086, 2016.

[25] J. Martin-Regalado, F. Prati, M. San Miguel, and N. Abraham, "Polarization properties of vertical-cavity surface-emitting lasers," IEEE Journal of Quantum Electronics, vol. 33, no. 5, pp. 765-783, 1997.

[26] P. Pérez, A. Valle, and L. Pesquera, "Polarization-resolved characterization of long-wavelength vertical-cavity surface-emitting laser parameters," J. Opt. Soc. Amer. B, vol. 31, no. 11, pp. 2574-2580, 2014.

[27] F. Denis-le Coarer, A. Quirce, P. Perez, A. Valle, L. Pesquera, M. Sciamanna, H. Thienpont, and K. Panajotov, "Injection locking and polarization switching bistability in a $1550 \mathrm{~nm}$-vcsel subject to parallel optical injection," IEEE Journal of Selected Topics in Quantum Electronics, 2017.

[28] S. P. Ó Dúill, P. M. Anandarajah, R. Zhou, and L. P. Barry, "Numerical investigation into the injection-locking phenomena of gain switched lasers for optical frequency comb generation," Applied Physics Letters, vol. 106, no. 21, p. 211105, 2015.

[29] P. Pérez, A. Valle, I. Noriega, and L. Pesquera, "Measurement of the intrinsic parameters of single-mode VCSELs," Journal of Lightwave Technology, vol. 32, no. 8, pp. 1601-1607, 2014.

[30] D. Wiedenmann, R. King, C. Jung, R. Jager, R. Michalzik, P. Schnitzer, M. Kicherer, and K. J. Ebeling, "Design and analysis of single-mode oxidized vcsels for high-speed optical interconnects," IEEE Journal of Selected Topics in Quantum Electronics, vol. 5, no. 3, pp. 503-511, May 1999.

[31] K. Panajotov, B. Nagler, G. Verschaffelt, A. Georgievski, H. Thienpont, J. Danckaert, and I. Veretennicoff, "Impact of in-plane anisotropic strain on the polarization behavior of vertical-cavity surface-emitting lasers," Applied Physics Letters, vol. 77, no. 11, pp. 1590-1592, 2000.

[32] T. Pusch, M. Lindemann, N. Gerhardt, M. Hofmann, and R. Michalzik, "Vertical-cavity surface-emitting lasers with birefringence splitting above 250 ghz," Electronics Letters, vol. 51, no. 20, pp. 1600-1602, 2015.

[33] M. AlMulla, "Optical double-locked semiconductor lasers," Results in Physics, vol. 9, pp. 63-70, 2018.

[34] B. Jerez, P. Martín-Mateos, E. Prior, C. de Dios, and P. Acedo, "Dual optical frequency comb architecture with capabilities from visible to mid-infrared," Opt. Express, vol. 24, no. 13, pp. 14986-14994, Jun 2016. [Online]. Available: http://www.opticsexpress.org/abstract.cfm? URI=oe-24-13-14986

[35] S. Chandran, S. Mahon, A. A. Ruth, J. Braddell, and M. Gutiérrez, "Cavity-enhanced absorption detection of $\mathrm{h} 2 \mathrm{~s}$ in the near-infrared using a gain-switched frequency comb laser," Applied Physics B, vol. 124, no. 4, p. 63, 2018.

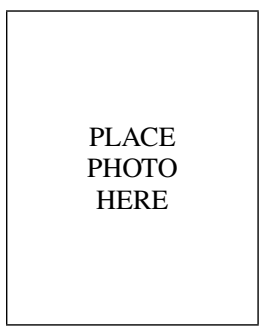

Ana Quirce received the Licenciada en Física (M.Sc.) degree and the Ph.D. degree in sciences, technologies, and computing from the University of Cantabria, Santander, Spain, in 2008 and 2012, respectively, where she was studying the dynamics of the polarization and transverse modes of vertical cavity surface-emitting lasers (VCSELs) subject to optical injection. She is currently a PostDoctoral Fellow from the Research FoundationFlanders (FWO) with the Vrije Universiteit Brussel, Brussels, Belgium. Her current research interests are in the areas of dynamics of VCSELs, optical injection effects in semiconductor lasers, optical feedback, optical frequency combs, and photonic integrated circuits.

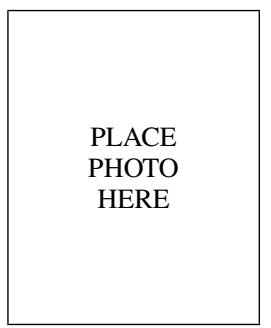

thesis and detection.
Cristina de Dios received the Doctorate degree in 2010 for her work in ultrafast pulsed diode lasers and nonlinear pulse compression at the Universidad Carlos III de Madrid, Spain. Currently, she is an associate professor at the Electronics Technology Department and a member of the Optoelectronics and Laser Technology Group in this same university. Her research interests are optical frequency comb generation techniques, pulsed semiconductor laser sources, nonlinear optical phenomena and subterahertz and millimeter wave photonic signal syn-

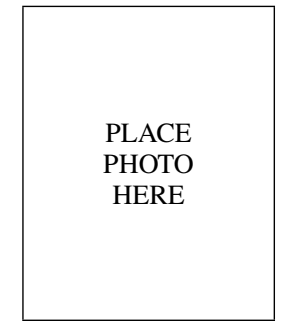

semiconductor lasers.
Angel Valle received the M. Sc. and Ph.D. degree in Physics from the Universidad de Cantabria, Spain, in 1988 and 1993, respectively. During 1994 and 1995, he was a postdoctoral fellow at the School of Electronic and Electrical Engineering at the University of Bath, England. In 1996 he joined the Instituto de Física de Cantabria (CSIC-UC). Since 1998 he has been lecturer at the Departamento de Física Moderna at the University of Cantabria, Spain. His research interests are in the areas of vertical-cavity surfaceemitting lasers, noise and nonlinear dynamics of

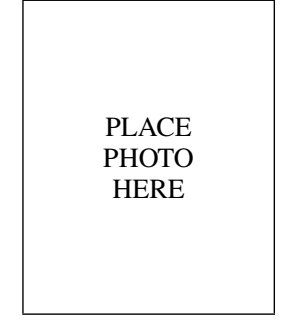

Pablo Acedo (M00) received the Doctorate (Hons.) degree from the Universidad Carlos III de Madrid, Leganes, Spain, in 2000. His doctoral work included the development of the first two color laser system based on Mid-IR sources for a stellarator fusion device (Stellarator TJ-II) and the first twocolor Nd:YAG system for a fusion device (Tokamak C-Mod). In 2002, he was appointed an Assistant Professor by the Universidad Carlos III de Madrid. During the last years, he has been very active in the development of advanced spectroscopy techniques, mainly based on multiheterodyne architectures using dual-optical frequency combs, with applications in fields like gas spectroscopy, environmental applications and, especially, in biomedical applications. 